\title{
OPTICAL BISTABILITY INDUCED BY ADDITIVE COLORED NOISE IN THE EXCITON-BIEXCITON SYSTEM
}

\author{
I.I. GonTyiA \\ Department of Physics, "Transilvania" University \\ Bul. Eroilor nr. 29 2200-Braşov, România \\ AND A.H. RotaRU \\ Department of Physics, Moldavian State University \\ Chişinău, Republica Moldova
}

(Received August 8, 1994; revised version December 22, 1994)

This paper predicts optical bimodality induced by external additive noise with a finite bandwidth, in the exciton-biexciton system.

PACS numbers: $05.40 .+\mathrm{j}, 05.70 . \mathrm{Fh}, 42.65 . \mathrm{Pc}_{\mathrm{C}}$

\section{Introduction}

An increasing attention has been paid lately to the noise-induced transitions arising in nonlinear systems far from thermodynamic equilibrium [1-12]. It is known that the noise in nonlinear systems has not only disorganizing effects but under certain conditions leads also to the appearance of nonequilibrium phase transitions that have no deterministic analogues [9]. The optical bistable system with a medium of two-level atoms is one of the systems in which the noise-induced transitions have been predicted; the external multiplicative white noise can induce optical bistability (OB) [7-9] and multistability for high values of noise intensity [10]. For the same system the colored noise influence on the $O B$ has also been studied, revealing that optical bimodality can arise from the multiplicative colored noise, as well as due to the additive colored noise [11].

The theoretical study and experimental observation of the OB phenomenon in semiconductors present a large interest owing to its possibility of practical applications. Many experimental results have demonstrated the existence of $O B$ in different types of semiconductors [13-15]. Theoretically, the OB in semiconductors has also been investigated in a series of works $[7,16-18]$. 
The effects of the noise upon the OB in semiconductors have less been dealt with and that is what we propose to do in this paper. Starting from a Fokker-Planck equation (FPE) deduced by Kus et al. in Ref. [12] we analyzed the influence of the incoming laser light on $\mathrm{OB}$ in the exciton-biexciton system.

\section{OB in the exciton-biexciton system}

A semiconductor interacting with an electromagnetic field in the vicinity of the two-photon biexciton resonance can be described as an idealized system involving two types of quasiparticles: excitons and biexcitons. Photons of the same impulse can excitate the excitons from the ground state of a crystal and convert the excitons into biexcitons. Because the quasiparticles behave as bosons we can write the Hamiltonian of the crystal in the form

$$
H_{\text {matter }}=\hbar \omega_{\text {ex }} a^{+} a+\hbar \omega_{\text {biex }} b^{+} b \text {, }
$$

where $a^{+}(a)$ and $b^{+}(b)$ are the creation (annihilation) operators for the excitons and biexcitons, respectively, and $\hbar \omega_{\text {ex }}$ and $\hbar \omega_{\text {biex }}$ are their corresponding transitions energies. We have considered a single coherent mode for the excitons and biexcitons.

The interaction Hamiltonian of excitons and biexcitons with the laser field, in the rotating wave and electric dipole approximations, is given by [17]

$$
H_{\mathrm{int}}=-\hbar g\left(E^{-} a+a^{+} E^{+}\right)-\hbar \sigma g\left(a^{+} b E^{-}+E^{+} b^{+} a\right),
$$

where $g$ is the coupling constant of the field with the exciton, $\sigma$ is the optical convertion constant of the excitons in biexcitons, $E^{+}\left(E^{-}\right)$is the component of electric field with positive (negative) frequency.

The full Hamiltonian of semiconductor interacting with a laser beam is

$$
H=H_{\text {matter }}+H_{\text {int }} \text {. }
$$

Using (3), we obtain the Heisenberg equations of motion for the operators $a$ and $b$

$$
\begin{aligned}
& \frac{\mathrm{d} a}{\mathrm{~d} t}=-\mathrm{i} \omega_{\mathrm{ex}} a+\mathrm{i} g E^{+}+\mathrm{i} \sigma g E^{-} b-\gamma_{\mathrm{ex}} a, \\
& \frac{\mathrm{d} b}{\mathrm{~d} t}=-\mathrm{i} \omega_{\mathrm{biex}} b+\mathrm{i} \sigma g E^{+}-\gamma_{\mathrm{biex}} b,
\end{aligned}
$$

where we have added the phenomenological relaxation rates $\gamma_{\text {ex }}$ and $\gamma_{\text {biex }}$ for the exciton and biexcitons, respectively.

We consider the electric field to be classical, described by Maxwell's equation

$$
\frac{\partial^{2} E^{+}}{\partial t^{2}}-\frac{1}{c^{2}} \frac{\partial^{2} E^{+}}{\partial t^{2}}=4 \pi \frac{\partial Q^{+}}{\partial t},
$$

where $Q^{+}=\hbar g\left(a+\sigma a^{+} b\right)$ is the polarization in the material and its form was deduced from the Hamiltonian of interaction. 
The solutions of Eqs. (4)-(5) are supposed to be the product of slowly varying envelopes and rapidly oscillating components with carrier frequency $\omega$ and wave vector $k$

$$
\begin{aligned}
& a=A(z, t) \exp (\mathrm{i} k z-\mathrm{i} \omega t), \quad b=B(z, t) \exp (2 \mathrm{i} k z-2 \mathrm{i} \omega t), \\
& E^{+}=e^{+}(z, t) \exp (\mathrm{i} k z-\mathrm{i} \omega t) .
\end{aligned}
$$

Replacing the relations (6) into Eqs. (4) and (5) and using the slowly varing approximation [19]

we obtain

$$
\left|\frac{\partial e^{+}}{\partial t}\right| \ll \omega\left|e^{+}\right|, \quad\left|\frac{\partial e^{+}}{\partial z}\right| \ll k\left|e^{+}\right|,
$$

$$
\begin{aligned}
& \frac{\mathrm{d} A}{\mathrm{~d} t}=\mathrm{i}\left(\omega-\omega_{\mathrm{ex}}\right) A-\gamma_{\mathrm{ex}} A-\mathrm{i} g e^{+}-\mathrm{i} \sigma g B e^{-}, \\
& \frac{\mathrm{d} B}{\mathrm{~d} t}=\mathrm{i}\left(2 \omega-\omega_{\mathrm{biex}}\right) B-\gamma_{\mathrm{biex}} B+\mathrm{i} g \sigma e^{-} A, \\
& 2 \mathrm{i} k c^{2} \frac{\partial e^{+}}{\partial z}+2 \mathrm{i} \omega \frac{\partial e^{+}}{\partial t}=4 \pi \hbar \omega^{2} g\left(A+\sigma A^{+} B\right) .
\end{aligned}
$$

Supposing that $\gamma_{\text {ex }}$ and $\gamma_{\text {biex }} \gg 1$ we can adiabatically eliminate the excitonic and biexcitonic variables by putting the time derivative in (7a) and (7b) to zero. For exact resonance we have

$$
A=-\frac{\mathrm{i} g \gamma_{\text {biex }} e^{+}}{\gamma_{\text {ex }} \gamma_{\text {biex }}+\sigma^{2} g^{2} e^{+} e^{-}}, \quad B=-\frac{g^{2} \sigma e^{+} e^{-}}{\gamma_{\mathrm{ex}} \gamma_{\mathrm{biex}}+\sigma^{2} g^{2} e^{+} e^{-}} .
$$

We substitute (8) into (7c) and we take $e^{+}=\varepsilon \mathrm{e}^{-\mathrm{i} \varphi}$. Neglecting the phase variation we finally obtain an equation for the field amplitude alone

$$
\frac{\omega}{k c^{2}} \frac{\partial \varepsilon}{\partial \iota}+\frac{\partial \varepsilon}{\partial z}=\frac{\alpha}{2} \frac{\varepsilon\left(1+2 \varepsilon^{2} / \varepsilon_{g}^{2}\right)}{\left(1+\varepsilon^{2} / \varepsilon_{g}^{2}\right)^{2}},
$$

where

$$
\alpha=\frac{4 \pi \hbar g^{2} \omega^{2}}{c^{2} k \gamma_{\mathrm{ex}}}, \quad \varepsilon_{g}^{2}=\frac{\sigma^{2} g^{2}}{\gamma_{\mathrm{ex}} \gamma_{\mathrm{biex}}} .
$$

The semiconductor sample of the length $L$ is placed into a ring cavity. Two of the cavity mirrors have transmitting and reflecting coefficients $T$ and $R$, respectively, while the other two are assumed to be ideal. We use the well-known boundary conditions for the field amplitude to the ends of the sample, at points 0 and $L$

$$
\varepsilon(0)=\sqrt{T} E_{\mathrm{I}}+R \varepsilon(L), \quad E_{\mathrm{T}}=\sqrt{T} \varepsilon(L), \quad E_{\mathrm{R}}=\sqrt{R T} \varepsilon(L)+\sqrt{R} E_{\mathrm{I}},
$$

where $E_{\mathrm{I}}, E_{\mathrm{T}}$ and $E_{\mathrm{R}}$ are the amplitude of the incident field on the entrance mirror, the amplitude of the exciting field from the cavity, and that of the reflected field. From (9), in the mean field approximation [19] and with allowance for the boundary condition (10), we can easily get the equation for the temporal evolution of the field amplitude

$$
\frac{\mathrm{d} x}{\mathrm{~d} \tau}=y-x-2 c \frac{x\left(1+2 x^{2}\right)}{\left(1+x^{2}\right)^{2}},
$$


where $x$ and $y$ are the normalized entrance and exit field amplitudes, $\tau$ is the dimensionless time and $c$ is a control parameter in the optical bistability theory. These are determined by the following expressions:

$$
x=\frac{\sigma g}{\sqrt{\gamma_{\mathrm{ex}} \gamma_{\mathrm{biex}} T}} E_{\mathrm{T}}, \quad y=\frac{\sigma g}{\sqrt{\gamma_{\mathrm{ex}} \gamma_{\mathrm{biex}} T}} E_{\mathrm{I}}, \quad \tau=\frac{\omega L}{c^{2} k} t, \quad c=\frac{\alpha l}{2 T} .
$$

In the stationary case Eq. (11) yields the equation obtained and studied in Ref. [18]

$$
y=x\left[1+\frac{2 c\left(1+2 x^{2}\right)}{\left(1+x^{2}\right)^{2}}\right]
$$

exhibiting a bistable behavior for $c>54 / 17$ (Fig. 1).

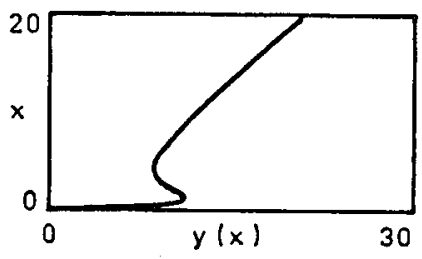

Fig. 1. Steady-state curve representing Eq. (13) with $c=10$.

\section{External additive colored noise-induced transitions}

In real conditions, however, the problem under consideration has different sources of noises, both additive and multiplicative noises. We will consider below the additive noise, taking as the main source of the noise the fluctuation of the amplitude of the incoming laser beam, characteristics of which can be controlled fairly well in experiments. The total driving electric field amplitude $y(t)$ can be written as a sum of two parts: a constant coherent part $y_{0}$ and a small fluctuating part $\xi(t)$, which we will consider to be an Orstein-Ulenbeck (O-U) process with the mean value zero and the correlation function

$$
\left\langle\xi(t) \xi\left(t^{\prime}\right)\right\rangle=a \mathrm{e}^{-\left(t-t^{\prime}\right) / b}
$$

where "a" measures the dimensionless intensity of the noise and $b=\gamma \tau_{c}$ is the coherence time $\tau_{\mathrm{c}}$ of the laser light expressed in the cavity linewidth $\gamma$. It can immediately be verified that for $b \rightarrow 0$ with $D=a b=$ const, the stochastic O-U process tends to the white noise limit; $\left\langle\xi_{\mathrm{w}}(t)\right\rangle=0$ and $\left\langle\xi_{\mathrm{w}}(t) \xi_{\mathrm{w}}\left(t^{\prime}\right)\right\rangle=2 D \delta\left(t-t^{\prime}\right)$.

Including the fluctuations of the incoming field amplitude, having the property (14), Eq. (11) becomes a Langevin equation with an additive $\mathrm{O}-\mathrm{U}$ stochastic process

$$
\frac{\mathrm{d} x}{\mathrm{~d} \tau}=y_{0}-x\left[1+\frac{2 c\left(1+2 x^{2}\right)}{\left(1+x^{2}\right)^{2}}\right]+\xi(t)
$$

For evaluating the influence of the additive colored noise on the $O B$ in the exciton-biexciton system, we will use the one-dimensional FPE deduced in Ref. [12]

$$
\frac{\partial P(x, \ell)}{\partial t}=-\frac{\partial}{\partial x}[F(x) P(x)]+D \frac{\partial^{2}}{\partial x^{2}}[K(x) P(x, \imath)],
$$


where

$$
F(x)=y_{0}-x\left[1+\frac{2 c\left(1+2 x^{2}\right)}{\left(1+x^{2}\right)^{2}}\right],
$$

$D=a b$ and $K(x)=1+b F^{\prime}(x), F^{\prime}(x)$ being the derivative of the function $F(x)$.

In order to obtain the above FPE it was supposed that $a<1$ and $b \leq 1$. Because the diffusion coefficient $K(x)$ must be positive, one gets $b \leq 1 /(1+2 c)$, which is consistent with $b<1$.

In the stationary case Eq. (16) yields

$$
\frac{\mathrm{d} J(x)}{\mathrm{d} x}=0, \quad J(x)=J_{0}=\text { const, }
$$

where $J(x)$ is the stationary probability flux

$$
J(x)=F(x) P(x)-D \frac{\mathrm{d}}{\mathrm{dx}}[K(x) P(x)] .
$$

Assuming the natural boundaries for the stochastic process we obtain the equation $J(x)=0$ for the stationary probability density $P(x)$, solution of which has the form $[20,21]$

$$
P(x)=N \exp \left[-\frac{1}{D} V(x)\right]
$$

where $N$ is the normalization constant and $V(x)$ is the stochastic potential

$$
V(x)=-\int \mathrm{d} x\left[\frac{F(x)}{1+b F^{\prime}(x)}\right]+a b \ln \left(1+b F^{\prime \prime}(x)\right) .
$$

In contrast to the deterministic case, in which the stationary states were obtained under the condition $F(x)=0$, stationary states in the stochastic case must be interpreted as the points of the system's state space at which the stationary probability density $P(x)$ takes on extremal values. The maximum points of the function $P(x)$ correspond to the most probable states of the system, and the minimum points to the least probable. The extrema of the $P(x)$ correspond to the extrema of the potential $V(x)$, the maximum points of $P(x)$ correspond to the minimum points of $V(x)$, and vice versa.

To determine the stationary states of the system we put $\mathrm{d} V / \mathrm{d} x$ to zero and as a result we get

$$
y_{0}=f(x, q, c)
$$

where

$$
f(x, q, c)=x+\frac{2 c x\left(1+2 x^{2}\right)}{\left(1+x^{2}\right)^{2}}-\frac{8 c q\left(x^{5}-5 x^{3}\right)}{\left(1+x^{2}\right)^{4}}
$$

and

$$
q=a b^{2}=D b
$$

We sce that the relation (21) which gives the most probable values of the stationary probability distribution depends on two coefficients $a$ and $b$ which characterize the laser amplitude fluctuations, and the parameter of bistability $c$. 
To analyze the behavior of the function $f(x, q, c)$, and hence the behavior of the stationary states of the system, we find the critical values of $c$ and $D$ from the conditions $f_{x}^{\prime}=f_{x x}^{\prime \prime}=0$, equivalent to the following system of equations:

$$
1-2 c \psi\left(x^{2}, q\right)=0, \quad \psi^{\prime}\left(x^{2}, q\right)=0,
$$

where

$$
\psi\left(x^{2}, q\right)=\frac{2 z^{4}-(12 q-1) z^{3}+(120 q-5) z^{5}-(60 q+5) z-1}{(1+z)^{5}}, \quad z=x^{2} .
$$

Taking into account that $b$ is given by the relation $b=1 /(1+2 c)$, (we have chosen the equality) we can find the expressions for the parameters $c$ and $D$

$$
\begin{aligned}
& c(z)=\frac{(1+z)^{5}\left(12 z^{3}-198 z^{2}+240 z-30\right)}{2\left(12 z^{7}-228 z^{6}-90 z^{5}+270 z^{4}-312 z^{3}-552 z^{2}-90 z+30\right)}, \\
& D(z)=\frac{\left(z^{4}-3 z^{3}-9 z^{2}-5 z\right)[1+2 c(z)]}{12 z^{3}-198 z^{2}+240 z-30} .
\end{aligned}
$$

We considered only the nonnegative values of $c$ and $D$.

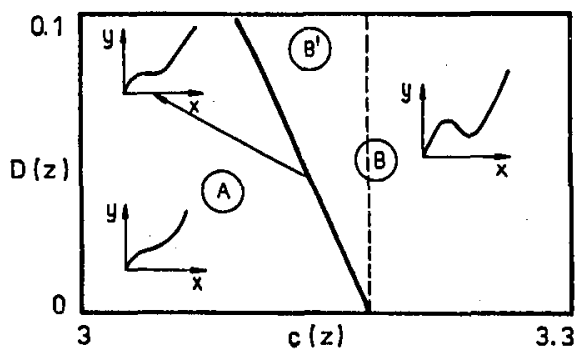

Fig. 2. The diagram of $D$ vs. $c$ describing the behavior of the stationary states of the system.

Formulas (23) specify the parametric equation of the separatrix in the $(c, D)$ plane (Fig. 2.), which divides the range of the variations of the parameters into two subsets $A$ and $B$, in which the behavior of the function $f(x, q, c)$, and hence the dependence of the stationary states of the system on the amplitude $y_{0}$ of the incident field, is markedly different. For $(c, D) \in A$, the stationary probability density $P(x)$ is unimodal and the system possesses a single stable stationary state. When the point crosses the boundary, separating $A$ and $B$, bistability appears. Depending on the amplitude $y_{0}$ of the incident field, the stationary probability density $P(x)$ is either unimodal, or bimodal, that is, the system has either one stable stationary state or three stationary states, two of which are stable and one unstable. The points belonging to the subset $B^{\prime}$ included in $B$, contained between the critical curve and the dashed-line (Fig. 2), represent the points for which the system becomes bistable only due to the noise, the value of parameter $c$ being under the critical deterministic value $c=54 / 17$.

We have shown that the noise-induced transitions (bistability) can appear due to the additive colored noise in a bistable semiconductor system in the excitonbiexciton range of the spectrum. 


\section{References}

[1] J.M. Sancho, M. San Miguel, S.L. Kats, J.D. Gunton, Phys. Rev. A 26, 1589 (1982).

[2] K. Lindenberg, B.J. West, Physica A 119, 485 (1983).

[3] L.A. Lugiato, R.J. Horowicz, J. Opt. Soc. Am. B 2, 971 (1985).

[4] P. Hanggi, T.J. Mroczkowski, F. Moss, P.V.E. McClintock, Phys. Rev. A 32, 695 (1985).

[5] P. Hanggi, F. Marchesoni, P. Grigolini, Z. Phys. B 56, 333 (1984).

[6] R.F. Fox, Phys. Rev. A 33, 467 (1986); ibid. 34, 4525 (1986).

[7] H. Gibbs, Optical Bistability: Controlling Light with Light, Academic Press, New York 1985.

[8] A.R. Bulsara, W.C. Schive, R.F. Gragg, Phys. Lett A 68, 294 (1978).

[9] W. Horsthemke, R. Lefever, Noise-Induced Transitions Theory and Applications in Physics, Chemistry, and Biology, Springer-Verlag, Berlin 1984.

[10] A.E. Barbăroşie, I.I. Gonţia, Yu N. Nica, A.H. Rotaru, Zh. Eksp. Teor. Fiz. 104, 2655 (1993).

[11] P. Grigolini, L.A. Lugiato, R. Manella, P.V.E. McClintock, M. Merri, M. Pernigo, Phys. Rev. A 38, 1966 (1988).

[12] M. Kus, K. Wodkiewicz, J.A.C. Gallas, Phys. Rev. A 28, 314 (1983).

[13] H.M. Gibbs, S.L. Mc Call, T.N.C. Venkatesan, A.C. Grossand, A. Passner, W. Wiegmann, App. Phys. Lett. 35, 451 (1979).

[14] D.A.B. Miller, S.D. Smith, A.M. Johnston, Appl. Phys. Lett. 35, 658 (1979).

[15] H.M. Gibbs, S.S. Tanng, J.L. Jewell, D.A. Weinberger, K. Tai, A.C. Gossard, S.L. McCall, A. Passner, W. Wiegmann, Appl. Phys. Lett. 41, 221 (1982).

[16] C.C. Sung, C.M. Bowden, Phys. Rev. A 29, 1957 (1984); S.W. Koch, H. Hang, Phys. Rev. Lett. 46, 450 (1981); I. Abram, Phys. Rev. B 28, 4433 (1983).

[17] P.I. Hadji, Nonlinear Optical Processes in Exciton-Biexciton System in Semiconductors, Ştiinţa, Chişinău 1985 (in Russian).

[18] P.I. Hadji, G.D. Shibarshina, A.H. Rotaru, Optical Bistability in Coherent Systems of Excilons and Biexcitons in Semiconductors, Ştiința, Chişinău 1988 (in Russian).

[19] R. Bonifacio, L.A. Lugiato, Lett. Nuovo Cimento 21, 505 (1976); ibid. 21, 520 (1976).

[20] C.W. Gardiner, Handbook of Stochastic Methods, Springer Verlag, Berlin 1983.

[21] H. Risken, The Fokker Planck Equation. Method of Solution and Applications, Springer Verlag, Berlin 1984. 